\title{
Detección de la Violencia contra la Pareja por Profesionales de la Salud
}

\section{Screening for Intimate Partner Violence by Health Professionals}

\author{
Esther Álvarez López, Carolina Brito, Karin Arbach y Antonio Andrés-Pueyo \\ Universidad de Barcelona
}

\begin{abstract}
Resumen. La violencia contra la pareja (VCP) es fuente de malestar en la mujer que la sufre y a menudo se traduce en problemas somáticos, sociales y/o psicológicos. Los profesionales que trabajan en el ámbito de la salud frecuentemente atienden a estas mujeres en su práctica clínica, por lo que juegan un papel relevante en la detección y atención de la VCP. Este estudio explora la práctica de la detección mediante una encuesta administrada a una muestra de 346 profesionales de la medicina, psiquiatría y psicología de Cataluña. Se analiza su opinión sobre la detección, los métodos que emplean, las actuaciones que realizan y las dificultades con que se encuentran. Los resultados sugieren que la mayoría de los profesionales se encuentran con casos de VCP. Aunque consideran que la detección forma parte de sus funciones y exploran la VCP, no suelen hacerlo de forma rutinaria ni pautada, no la realizan en la primera visita ni con preguntas directas, sino que depende en gran medida de la presencia de indicios.
\end{abstract}

Palabras clave: Violencia contra la pareja, detección, víctimas, estudio descriptivo mediante encuesta.

\begin{abstract}
Intimate partner violence (IPV) is a source of distress for women who suffer it and often it cause somatic, social and psychological problems. Professionals working in health care facilities frequently attend these women in their clinical practice, so they play a relevant role in detection and care of IPV. The main aim in this study was to explore how physicians, psychiatrists and psychologists detect IPV in their practice. We lead a survey in a sample composed by 346 professionals of Catalonia. We analyze their opinion about detection task, the methods they use, the actions they take and, difficulties they usually find. Our results suggest that most professionals see IPV's cases in their practice. Although they consider screening as part of their duties, they do not make routine or structure screenings, neither they screen it in the first meeting, nor with direct questions. The screening depends significantly on the presence of IPV signs or indicators.

Keywords: Intimate partner violence, screening, victims, survey descriptive study.
\end{abstract}

\section{Introducción}

Para combatir la violencia contra la pareja (VCP) son indispensables tanto las intervenciones jurídico-

La correspondencia sobre este artículo debe enviarse al cuarto autor a la Universidad de Barcelona, Grupo de Estudios Avanzados en Violencia, Paseo Vall Hebron 17, 08035 Barcelona. E-mail: andrespueyo@ub.edu penales como las sociosanitarias. Asimismo muchos expertos coinciden en considerar que la mejor medida para actuar contra la violencia hacia las mujeres

Nota: Estudio financiado parcialmente por el Institut Català de les Dones (ICD07.003)

Agradecimiento: Nuestro agradecimiento a todos los y las profesionales que han participado en esta investigación haciéndonos llegar sus respuestas a la encuesta. 
es la prevención. La VCP está tan extendida que es frecuente considerarla de forma análoga a una pandemia (Ellsberg y Heise, 2005; Krug, Dahlberg, Mercy, Zwi y Lozano, 2002), y en este caso las estrategias preventivas más eficaces incluyen una de especial interés: la detección temprana. El cribado o "screening" de la VCP consiste en la identificación de situaciones consolidadas de VCP pero también permite identificar otras situaciones de violencia que sean incipientes o accidentales y que no están identificadas como VCP. Estas técnicas de cribado no son exclusivas de ningún profesional especializado en la lucha contra la violencia hacia las mujeres sino que deberían ser aplicadas por todos aquellos que atienden posibles víctimas en contextos como la asistencia primaria o la práctica clínica.

La importancia de los profesionales sanitarios en la lucha contra la VCP en España está recogida entre otros ámbitos en el legal ya que existe la obligación de poner en conocimiento de la autoridad judicial la existencia de lesiones ante la constatación de malos tratos, obligación que se cumple mediante la notificación al juzgado del parte judicial. La Ley Orgánica 1/2004 de 28 de diciembre, de Medidas de Protección Integral contra la Violencia de Género establece en su Título III, sobre Tutela institucional, en su artículo 32, sobre Planes de Colaboración que: «Los protocolos, además de referirse a los procedimientos a seguir, harán referencia expresa a las relaciones con la Administración de Justicia, en aquellos casos en que exista constatación o sospecha fundada de daños físicos o psíquicos ocasionados por estas agresiones o abusos». Esta sospecha fundada podría verse refrendada por los procedimientos de cribado. También el artículo 262 de la Ley de Enjuiciamiento Criminal señala que: «Los que por razón de sus cargos, profesiones u oficios tuvieren noticia de algún delito público, estarán obligados a denunciarlo inmediatamente al Ministerio Fiscal, al Tribunal competente, al Juez de instrucción y, en su defecto, al municipal o al funcionario de policía más próximo al sitio, si se tratare de un delito flagrante». Según establece la Ley de Enjuiciamiento Criminal, la Fiscalía podrá, con carácter previo a la acción judicial - antes de que llegue al Juez correspondiente la denuncia del delito y este emita su veredicto- ordenar la realización de prediligencias de investigación, para recabar más datos e información acerca de los hechos denunciados.

Los profesionales que desempeñan sus tareas en el ámbito sanitario (p.e. medicina, enfermería, psicología,...), juegan un rol crucial para la detección temprana e intervención en los casos de VCP. Este papel está directamente reconocido en la Ley Orgánica 1/2004 de 28 de diciembre, de Medidas de Protección Integral contra la Violencia de Género que, en congruencia con otras leyes, indica qué obligaciones tienen para con la ley.

Son conocidos los efectos de la VCP sobre la salud física, mental y social (Amor, Echeburúa, de Corral, Zubizarreta y Sarasua, 2002; Ellsberg, Jansen, Heise, Watts y Garcia-Moreno, 2008; Plazaola-Castaño y Ruiz Pérez, 2004; Ruiz Pérez y Plazaola Castaño, 2005) y cómo este tipo de violencia puede ser la causa de signos y síntomas susceptibles de ser mal diagnosticados si no se tiene en cuenta el contexto en donde se generan.

Por su cercanía con la ciudadanía, los profesionales de la salud pueden ser -y de hecho lo son en muchas ocasiones- los primeros receptores de las demandas de mujeres víctimas de VCP. Dichas demandas a menudo no son directas y la VCP se oculta tras quejas vagas o inespecíficas de tipo físico, psicológico y/o social. En este sentido, los profesionales de los ámbitos sanitario y social están en disposición de ayudar a las usuarias mediante una acción preventiva de primer orden (Andrés Pueyo y Álvarez López, 2006).

Asimismo, es habitual que mujeres de todas las edades acudan al sistema sanitario por variadas razones, lo que convierte estos servicios en observatorios privilegiados para la detección. Un estudio realizado por el equipo de Fernández Alonso en Castilla y León (Ilustre Colegio Oficial de Médicos de Madrid, 2006) indica que el 82,8\% de las mujeres que sufrieron maltrato habían consultado con su médico de familia por diferentes motivos en los dos años previos al diagnóstico de maltrato. También se ha contrastado en EEUU, que del total de las mujeres que acuden a los servicios de atención primaria por cualquier motivo, el $20 \%$ declara estar recibiendo maltratos en el momento de la visita (Lasheras Lozano y Pires Alcaide, 2003). Por último también 
indicar que el malestar lleva a muchas de estas mujeres a consultar y utilizar los servicios sanitarios con mayor frecuencia, especialmente atención primaria, urgencias, obstetricia y ginecología y salud mental (Comisión contra la Violencia de Género del Consejo Interterritorial del SNS, 2007).

La detección de la VCP por parte de profesionales de la salud, está dificultada por una serie de obstáculos (Elliot, Nerney, Jones y Friedmann, 2002) que pueden provenir tanto de la propia víctima, de los profesionales o de las instituciones y con frecuencia ni la una ni los otros son conscientes de su existencia y sus consecuencias (Lasheras Lozano y Pires Alcaide, 2003). Los valores, actitudes y prejuicios afectan a la percepción de la VCP (García Moreno, 2002) que usualmente es vista por los profesionales de la salud como un tema no-médico (Coll-Vinent, Echevarría, Rodríguez y Santiñá, 2007). Si se reconociera la VCP como un factor de riesgo para distintos problemas de salud sería más aceptable para los profesionales clínico-sanitarios considerarla en sus evaluaciones.

Diferentes investigaciones y la propia OMS (1998) indican la importancia del cribado sistemático y universal de la VCP, preferentemente realizado con protocolos específicos, por parte de los profesionales de la salud en el marco de un programa de prevención y detección. Dicha práctica facilitaría estos efectos: a) detección de casos que son opacos al sistema judicial, b) prevención de las consecuencias del maltrato y c) educación socio-sanitaria de las mujeres ante este fenómeno. En general, las investigaciones señalan que actualmente no se realiza este cribado de forma universal (Rodriguez, Bauer, McLoughlin y Grumbach, 1999; Stayton y Duncan, 2005).

La introducción de protocolos validados incrementa la capacidad de identificación de la VCP (Mc Farlane, Christoffel, Bateman, Miller y Bullock, 1991; Wiist y McFarlane, 1999). En ellos se recomienda preguntar directamente sobre el tema a las usuarias, aunque se aconseja también atender otros indicadores. Varios estudios muestran que un elevado porcentaje de mujeres están a favor de la detección desde el contexto sanitario (Ramsay, Richardson, Carter, Davidson y Feder, 2002) y de que se les pregunte directamente sobre la cuestión (Morrison, Allan y Grunfeld, 2000). El estudio realizado en Castilla y León (Ilustre Colegio Oficial de Médicos de Madrid, 2006) señala que a un $78 \%$ de las víctimas de maltrato y abuso no les hubiera importado que su médico de familia les hubiera preguntado por su situación, pero concluye que sólo un $7,9 \%$ de los profesionales pregunta directamente sobre la VCP. En diferentes guías dirigidas al personal sanitario, se recomienda abordar directamente con la usuaria el tema de la violencia (Guia per a l'abordatge del maltractament de gènere a l'atenció primària, 2003; Comisión contra la Violencia de Género del Consejo Interterritorial del SNS, 2007; O.M.S. y O.P.S., 1998).

Cabe destacar que hay ciertos desacuerdos en cuanto a la efectividad del cribado de VCP. En la revisión realizada por el equipo de Vives (2006), se concluye que "no existe suficiente evidencia para afirmar que las mujeres se benefician clínicamente de las pruebas de detección temprana del maltrato" y que la aplicación de dichas pruebas en España "proporcionaría resultados poco útiles". Ahora bien, se condicionan dichos resultados a la ausencia de estudios metodológicamente adecuados, a la baja aceptación de estas estrategias por parte de los profesionales y a la falta de inclusión de un sistema de evaluación sobre VCP entre las responsabilidades de las administraciones sanitarias.

Por nuestra parte, y de acuerdo con lo que indica la OMS (O.M.S. y O.P.S., 1998), consideramos que son precisas ciertas condiciones en la implementación del cribado universal y de los protocolos en el sistema sanitario para evitar una aplicación inadecuada de los mismos. Es importante que haya una formación previa de los profesionales. El adiestramiento permite actualizar conocimiento y mejorar las habilidades profesionales, a la par que propicia un cambio de actitud más favorable a la detección. Algunos estudios sugieren la necesidad de fomentar la formación y reforzar circunstancias ambientales para mejorar la práctica de la detección (Samuelson y Campbell, 2005; Stayton y Duncan, 2005).

La mayoría de las investigaciones acerca de la práctica de la detección provienen de países anglosajones y se centran básicamente en colectivos de medicina y/o enfermería y de forma aislada de psi- 
cología (Samuelson y Campbell, 2005). En nuestro entorno cercano tenemos conocimiento de algunos estudios realizados con profesionales de medicina y enfermería (Coll-Vinent et al., 2007; Ilustre Colegio Oficial de Médicos de Madrid, 2006; Tomás Vecina, Chanovas Borrás y Roqueta Egea, 2008), pero no nos constan con profesionales de otras disciplinas, lo que nos llevó a plantear la presente investigación.

El objetivo principal de este estudio es conocer si los profesionales del ámbito de la salud (medicina de familia, pediatría, ginecología, psiquiatría y psicología) exploran la presencia VCP en sus usuarias. Así mismo, nos interesa saber cuál es su opinión al respecto, qué métodos y estrategias utilizan en la detección y atención y qué dificultades y limitaciones encuentran. Por otra parte, se pretende analizar las diferencias existentes al respecto entre los diferentes colectivos de profesionales.

\section{Método}

\section{Muestra}

La muestra analizada es de 346 profesionales de Cataluña de los ámbitos de la Psicología Clínica y de las especialidades de Medicina de Familia, Ginecología, Pediatría y Psiquiatría. En la Tabla 1 se recoge la distribución de los participantes y algunas de sus características socio-demográficas (edad, sexo y años de ejercicio profesional). Por lo que se refiere al total de la muestra, el 29,6\% ejerce en el ámbito público exclusivamente, el $41,3 \%$ en el sector privado y el $29 \%$ en ambos o en instituciones concertadas. El 40,5\% de los participantes desarrollan su tarea profesional en la ciudad de Barcelona, el $52,2 \%$ en otros municipios y el 7,3\% en ambas localizaciones. Respecto a los usuarios que reciben, el $85,2 \%$ atiende a mujeres adultas, un $59,4 \%$ a varones adultos y un $46,5 \%$ a adolescentes (la mayoría no de forma exclusiva).

Se procedió a agrupar las especialidades de Medicina de Familia, Pediatría y Ginecología en una misma categoría ("Medicina"), dado que el número de sujetos en cada una de ellas era bajo.

\section{Instrumento}

Se ha diseñado un cuestionario ad hoc, de diez ítems de respuesta cerrada y un espacio para comentarios personales, denominado Detección de la Violencia contra la Pareja ICD-UB. En su construcción se tuvieron en cuenta algunos de los ítems que se habían formulado en estudios similares. El cuestionario fue accesible desde nuestra página web (www.ub.edu/geav).

\section{Procedimiento}

Para la obtención de la muestra se contactó con los Colegios Profesionales de Psicología y de Medicina de Cataluña, que ofrecieron su consentimiento para acceder a la base de datos de colegiados. Se descartó a aquellos profesionales que ejercían en servicios específicos de atención a la VCP y se respetó la distribución de género dada en cada profesión. De forma aleatoria se seleccionaron 1576

Tabla 1. Distribución de la muestra en función de la profesión, edad media, sexo y media de años de ejercicio profesional

\begin{tabular}{lllcll}
\hline Profesión & Especialidad & N & Edad media (ds) & \% mujeres & $\begin{array}{c}\text { Media años } \\
\text { ejerc. prof. (ds) }\end{array}$ \\
\hline Psicología & & 221 & $44,1(9,5)$ & $71,6 \%$ & $16,4(8,9)$ \\
\hline Medicina & de Familia & 66 & $43,8(6,3)$ & $62,1 \%$ & $18,1(7,2)$ \\
& Ginecología & 29 & $52,5(4,8)$ & $65,5 \%$ & $27,3(5,7)$ \\
& Pediatría & 12 & $54,8(4,5)$ & $75,0 \%$ & $29,0(7,1)$ \\
& Psiquiatría & 18 & $50,1(7,6)$ & $61,1 \%$ & $22,9(7,8)$ \\
\hline Total & & 346 & $45,4(8,9)$ & $68,8 \%$ & $18,3(8,9)$ \\
\hline
\end{tabular}


psicólogos clínicos, 200 psiquiatras, 600 médicos de familia, 200 ginecólogos y 200 pediatras, residentes en Cataluña.

Se envió por correo postal a los profesionales seleccionados un sobre que contenía el cuestionario impreso junto con otro sobre ya franqueado para que remitieran su respuesta. Por otra parte, en nuestra página web se creó un enlace, para acceder al cuestionario en su versión electrónica. En el impreso en papel del cuestionario, se informaba sobre la posibilidad de respuesta por vía electrónica. En ambos medios se explicaba que el cuestionario formaba parte de un estudio sobre las posibilidades de detección precoz de la violencia contra las mujeres en el ámbito familiar y de pareja y se invitaba a contestar las diez preguntas.

De los 2776 envíos realizados, 156 fueron devueltos por tener direcciones incompletas o erróneas. Se recibieron 368 cuestionarios cumplimentados (el $94 \%$ por correo electrónico y el $6 \%$ por vía electrónica). De todas las encuestas recibidas se comprobó su validez y la muestra quedó conformada por 346 sujetos (véase Tabla 1). El porcentaje de respuesta de los sujetos respecto al número de envíos realizados osciló entre un $6 \%$ en la especialidad de pediatría hasta un $14,5 \%$ en ginecología. En el colectivo de psicología respondió un 14\%, en medicina de familia un $11 \%$ y en psiquiatría un $9 \%$.

Para realizar el análisis de los datos recogidos se utilizó el paquete estadístico informático SSPS (versión 12.0). Se realizaron los análisis descriptivos pertinentes y la prueba de Chi-cuadrado $\left(\chi^{2}\right)$ para cada una de las variables del cuestionario en relación con otras variables (profesión, sexo, ámbito de trabajo, lugar de ejercicio de la profesión y conocimiento de la prevalencia de la VCP). La variable "Profesión" fue la que presentó el mayor número de relaciones estadísticamente significativas con el resto de variables por lo que se presentarán únicamente estos resultados. Por último, aun cuando no se analizarán los comentarios de las opciones abiertas, sí se hará mención de algunas de las respuestas.

\section{Resultados}

La Tabla 2 presenta, a modo de síntesis, los porcentajes en las respuestas para cada uno de los ítems en función de la profesión.

El cálculo de la prueba Chi-cuadrado $(\chi 2)$ para cada una de las variables del cuestionario en relación con la variable profesión, indica que existen algunas diferencias significativas en la distribución

Tabla 2. Porcentaje de respuesta en los ítems del ICD-UB en función de la profesión

Porcentaje de respuesta

\begin{tabular}{|c|c|c|c|c|}
\hline Ítem & Total & Psicología & Psiquiatría & Medicina \\
\hline \multicolumn{5}{|c|}{ 1. ¿Se encuentra con casos de VCP en su práctica profesional? } \\
\hline Siempre o casi siempre & 2,0 & 2,3 & 0,0 & 1,9 \\
\hline A veces & 70,3 & 67,4 & 77,8 & 74,8 \\
\hline Nunca o casi nunca & 27,7 & 30,3 & 22,2 & 23,4 \\
\hline \multicolumn{5}{|l|}{ 2. ¿Explora la presencia de VCP? } \\
\hline Sí & 68,5 & 74,1 & 83,3 & 54,7 \\
\hline \multicolumn{5}{|c|}{ 3. ¿Cree que la detección de VCP se incluye en sus funciones actuales? } \\
\hline Sí & 31,6 & 29,3 & 38,9 & 34,9 \\
\hline Sí, sólo si hay indicios & 59,9 & 61,9 & 50,0 & 57,5 \\
\hline No & 8,6 & 8,8 & 11,1 & 7,5 \\
\hline \multicolumn{5}{|c|}{ 4. En relación a la detección de VCP usted cree que sería conveniente explorar ... } \\
\hline Todas las mujeres en la primera visita & 5,2 & 5,9 & 5,6 & 3,7 \\
\hline Todas después de primera visita & 9,2 & 9,5 & 16,7 & 7,5 \\
\hline Sólo si se sospecha VCP & 78,3 & 78,7 & 72,2 & 78,5 \\
\hline Sólo si usuaria expone el tema & 9,2 & 9,5 & 5,6 & 9,3 \\
\hline Nunca es conveniente explorarlo & 0,6 & 0,5 & 0,0 & 0,9 \\
\hline
\end{tabular}


Tabla 2. Porcentaje de respuesta en los ítems del ICD-UB en función de la profesión (continuación)

Porcentaje de respuesta

\begin{tabular}{|c|c|c|c|c|}
\hline Ítem & Total & Psicología & Psiquiatría & Medicina \\
\hline \multicolumn{5}{|c|}{ 5. ¿Qué método escogería para detectar la VCP en las usuarias? } \\
\hline Preguntar directamente & 13,4 & 16,4 & 5,6 & 8,5 \\
\hline Preguntar por malestar & 37,6 & 36,5 & 22,2 & 42,5 \\
\hline Preguntar indirectamente & 70,8 & 75,3 & 88,9 & 58,5 \\
\hline Otras fuentes & 7,6 & 9,1 & 11,1 & 3,8 \\
\hline Inferir por indicios & 12,8 & 13,2 & 5,6 & 13,2 \\
\hline \multicolumn{5}{|c|}{ 6. ¿Ha recibido formación especializada en temas de VCP? } \\
\hline Sí & 27,3 & 29,9 & 5,9 & 25,5 \\
\hline No, pero tengo interés por & & & & \\
\hline sobre el tema & 43,3 & 48,1 & 58,8 & 31,1 \\
\hline No & 29,4 & 22,0 & 35,3 & 43,4 \\
\hline \multicolumn{5}{|c|}{ 7. ¿Utiliza un protocolo específico para la detección de la VCP? } \\
\hline Sí & 8,4 & 6,8 & 0,0 & 13,1 \\
\hline \multicolumn{5}{|c|}{ 8. En su opinión ¿en su trabajo existen obstáculos para la detección de VCP? } \\
\hline Sí & 63,9 & 53,9 & 55,6 & 85,8 \\
\hline Usuaria no quiere revelar & 37,9 & 33,0 & 44,4 & 46,7 \\
\hline Puede molestarle & 14,5 & 10,9 & 11,1 & 22,4 \\
\hline Falta de entrenamiento & 29,5 & 19,5 & 11,1 & 53,3 \\
\hline Sentimiento intrusión & 11,6 & 5,9 & 21,5 & 22,2 \\
\hline Falta de tiempo & 24,6 & 5,9 & 22,2 & 63,6 \\
\hline Falta continuidad & 12,1 & 11,8 & 27,8 & 10,3 \\
\hline Falta de protocolo & 19,4 & 16,3 & 22,2 & 25,2 \\
\hline Otros & 5,5 & 6,3 & 0,0 & 4,7 \\
\hline
\end{tabular}

9. Por favor, indique con qué frecuencia usa Ud. Las siguientes intervenciones ante una víctima de VCP

9.1. Registro la declaración en su historia clínica

\begin{tabular}{|c|c|c|c|c|}
\hline Nunca o casi nunca & 6,8 & 9,2 & 0,0 & 3,2 \\
\hline A veces & 9,2 & 9,2 & 0,0 & 10,6 \\
\hline Frecuentemente & 11,6 & 9,2 & 25,0 & 13,8 \\
\hline Siempre o casi siempre & 72,4 & 72,3 & 75,0 & 72,3 \\
\hline
\end{tabular}

9.2. Ofrezco a la usuaria información específica de VCP

$\begin{array}{lrrrr}\text { Nunca o casi nunca } & 7,5 & 6,7 & 6,7 & 9,3 \\ \text { A veces } & 25,4 & 24,7 & 26,7 & 26,7 \\ \text { Frecuentemente } & 19,4 & 19,1 & 26,7 & 18,6\end{array}$

9.3. Derivo el caso a otro profesional

Nunca o casi nunca $\quad 15,9 \quad 21,2$

A veces

Frecuentemente 16

Siempre o casi siempre

16,2

$21,2 \quad 14,3 \quad 6,5$

$\begin{array}{lll}47,9 & 57,1 & 35,9\end{array}$

$12,1 \quad 14,3 \quad 23,9$

$\begin{array}{lll}23,6 & 18,8 & 14,3\end{array}$

9.4. Hago intervenciones precisas según lo pautado en un protocolo

Nunca o casi nunca 63,0

A veces 14,3

64,1

$64,1 \quad 69,2 \quad 60,0$

Frecuentemente

$13,9+13,4$

$15,4 \quad 10,7$

$13,4 \quad 15,4 \quad 14,7$

Siempre o casi siempre

8,7

0,0

14,7

9.5. Propongo a la usuaria que denuncie

Nunca o casi nunca 11,3

A veces $\quad 39,8$

Frecuentemente $\quad 19,0$

Siempre o casi siempre $\quad 29,9$

\begin{tabular}{|c|c|c|}
\hline 15,9 & 0,0 & 4,5 \\
\hline 42,4 & 66,7 & 30,3 \\
\hline 17,1 & 13,3 & 23,6 \\
\hline 24,7 & 20,0 & 41,6 \\
\hline
\end{tabular}


Tabla 2. Porcentaje de respuesta en los ítems del ICD-UB en función de la profesión (continuación)

Porcentaje de respuesta

\begin{tabular}{|c|c|c|c|c|}
\hline Ítem & Total & Psicología & Psiquiatría & Medicina \\
\hline \multicolumn{5}{|l|}{ 9.6. Intervengo de otra forma } \\
\hline Nunca o casi nunca & 28,6 & 21,3 & 75,0 & 42,3 \\
\hline A veces & 9,5 & 9,3 & 0,0 & 11,5 \\
\hline Frecuentemente & 8,6 & 8,0 & 25,0 & 7,7 \\
\hline Siempre o casi siempre & 27,6 & 32,0 & 0,0 & 19,2 \\
\hline Sin frecuencia & 25,7 & 29,3 & 0,0 & 19,2 \\
\hline \multicolumn{5}{|c|}{ 10. ¿Qué porcentaje de mujeres cree que sufren VCP cada año en Cataluña? } \\
\hline$<5 \%$ & 10,5 & 7,7 & 6,3 & 16,8 \\
\hline $5-15 \%$ & 55,3 & 53,1 & 62,5 & 58,4 \\
\hline $15-30 \%$ & 30,4 & 34,7 & 25,0 & 22,8 \\
\hline $30-45 \%$ & 2,6 & 3,1 & 0,0 & 2,0 \\
\hline$>45 \%$ & 1,3 & 1,5 & 6,3 & 0,0 \\
\hline
\end{tabular}

de respuestas entre los tres grupos. Concretamente, en el ítem 2 “Explora la presencia de VCP?” (14,3; $p \leq 0,001)$, en el ítem 5 “¿Qué método escogería para detectar la VCP en las usuarias?" para la alternativa "Preguntar indirectamente" $(12,8 ; p \leq 0,01)$, en el ítem 6 "¿Ha recibido formación especializada en temas de VCP?" $(20,5 ; p \leq 0,001)$, en el ítem 8 "En su opinión ¿en su trabajo existen obstáculos para la detección de VCP?" $(32,1 ; p \leq 0,001)$ y para las alternativas de respuesta de este ítem "La usuaria no quiere revelar" $(6,1 ; p \leq 0,05)$, "Puede molestar a la usuaria" $(8,0 ; p \leq 0,05)$, "Falta de entrenamiento" (42,7; $p \leq 0,001)$, "Sentimiento de intrusión" (19,3; $p \leq 0,001)$, "Falta de tiempo" (129,4; $p \leq 0,001)$. También encontramos diferencias significativas en el ítem 9 "Por favor, indique con qué frecuencia usa usted las siguientes intervenciones ante una víctima de VCP" para las alternativas de respuesta "Derivo el caso a otro profesional" $(21,7 ; p \leq 0,001) \mathrm{y}$ "Propongo a la usuaria que denuncie" (21,2; $p \leq 0,01)$.

\section{Discusión}

Los organismos internacionales indican la necesidad de aplicar estrategias de cribado sistemático para la detección de la VCP por parte de profesionales del ámbito de la salud. A pesar de ello, los estudios muestran que la realidad está lejos del cumplimiento de estas indicaciones. No obstante la mayo- ría de los estudios con los que contamos provienen del mundo anglosajón y del ámbito de la medicina y no son tantos los que ofrecen datos acerca de otras disciplinas y en nuestro entorno cercano. En el presente estudio hemos analizado la práctica de la detección de médicos, psiquiatras y psicólogos de Cataluña y sus opiniones al respecto.

En líneas generales, se puede observar que un $72,3 \%$ de los profesionales encuestados, a pesar de no trabajar en servicios específicos, se enfrentan a casos de VCP, cifra superior a la obtenida por Coll-Vinent et al. (2007) que lo situaban en el $41,2 \%$. Por tanto, se constata que esta es una realidad que se hace visible y tiene presencia en las consultas del ámbito de salud. Se comprueba que existe cierta sensibilidad por parte de los profesionales para explorar la presencia de VCP. El 68,5\% dice explorarla, independientemente de la forma y el momento, destacando especialmente el colectivo de psiquiatras $(83,3 \%)$, aunque no existen diferencias significativas entre las categorías profesionales.

Buena parte de los profesionales consideran que tienen cierta responsabilidad en la detección, aunque un $8,8 \%$ de los encuestados no cree que la detección se incluya entre sus funciones profesionales actuales. Este dato es similar al obtenido por Samuelson y Campbell (2005), según el cual el 95\% de los psicólogos en EEUU cree que es su responsabilidad asistir a las víctimas de VCP. No obstante, llevar a cabo la detección parece que depende en gran medi- 
da de la presencia de signos. En nuestro estudio, el $60 \%$ considera la detección como función propia sólo si hay sospechas de la presencia de VCP y el $78 \%$ cree que es conveniente la exploración únicamente en casos que presenten indicios. Sólo un 5,2\% consideró que sería adecuado explorar a todas las mujeres en la primera visita. Estas cifras son similares a las de otros estudios (Rodriguez et al., 1999; Stayton y Duncan, 2005), y nos indican, que la realidad de la práctica profesional está lejos del cribado universal al que instan instituciones y organizaciones no gubernamentales.

Sin embargo, observamos grandes diferencias respecto a los estudios de Coll-Vinent et al. (2007) según el cual un 91,5\% de los profesionales encuestados están de acuerdo con incluir de forma sistemática ítems específicos de cribado en la anamnesis en todos sus pacientes y los de Tomás Vecina et al. (2008) que indica que el $93 \%$ de los servicios encuestados tienen protocolizada la atención a víctimas. Cabe destacar que estas investigaciones se llevaron a cabo en contextos (hospitalario y de urgencias respectivamente) en los que, en general, ha habido una mayor implementación de guías y protocolos de actuación.

Observamos que tampoco se siguen las recomendaciones oficiales (Guia per a l'abordatge del maltractament de gènere a l'atenció primària, 2003; Comisión contra la Violencia de Género del Consejo Interterritorial del SNS, 2007; O.M.S. y O.P.S., 1998) y las preferencias de las usuarias (Ilustre Colegio Oficial de Médicos de Madrid, 2006) en lo que respecta al método de detección de VCP. Sólo el $13,4 \%$ refiere que la estrategia más adecuada es preguntar directamente a la usuaria si sufre VCP y sin embargo un $71 \%$ se decantan por preguntar de manera indirecta (sobre la relación de pareja, su satisfacción, etc.). Hay que destacar que, hay diferencias significativas entre colectivos, siendo los médicos los que menos indican la opción de preguntar de forma indirecta $(58,5 \%)$ frente a los psiquiatras que la señalan más $(88,9 \%)$.

Es probable que esta falta de precisión en la labor técnica, esté en parte asociada a dos cuestiones que, de hecho se relacionan entre sí. Por una parte, la falta de formación adecuada y/o actualizada (Cann, Withnell, Shakespeare, Doll y Thomas,
2001; Coll-Vinent et al., 2007; Stayton y Duncan, 2005; Tomás Vecina et al., 2008) y por otra, el escaso uso de protocolos de detección. De los profesionales encuestados, menos de un tercio $(27,3 \%)$ ha recibido formación especializada, aunque un 43,3\% se considera autodidacta (se interesa por leer bibliografía específica). El colectivo de psicólogos es el que está más instruido $(29,9 \%)$ frente al de psiquiatras $(5,9 \%)$ que, sin embargo, son más autodidactas que el resto $(58,8 \%)$, siendo el grupo de los médicos el menos formado respecto a los demás $(43,4 \%)$. La inclusión en los programas formativos de contenidos acerca de la detección temprana, posibilita a los profesionales conocer los avances en la materia y favorece un cambio de actitud en pro de los beneficios que pueda aportar. No obstante, se hace hincapié en la necesidad de que dicha formación sea previa a la implementación de las técnicas de cribado.

Aunque los niveles de formación especializada son bajos, todavía lo es menos el uso de protocolos específicos para la detección de VCP (sólo los emplea un $8,4 \%$ ). En este sentido hay que apelar al esfuerzo de organismos e instituciones para editar, difundir e implementar guías específicas. No obstante, debemos plantearnos el peso específico que tienen diferentes elementos (formación especializada, organización institucional) en el uso real de los protocolos para la buena praxis.

Es de suma importancia revisar los factores que se relacionan con las bajas tasas de detección (Elliot et al., 2002). Según nuestros datos, casi dos terceras partes de los profesionales (64\%) indican la existencia de obstáculos para llevar a cabo la detección. Entre estos, muchos consideraron que los obstáculos provienen más de la propia víctima -por ej., no querer revelar la información $(37,9 \%)$, sentirse molesta (14,5\%)- que de los propios profesionales -por ej., falta de entrenamiento específico $(29,5 \%)$, sentirse intrusivos $(11,6 \%)$ - o de la organización institucional -por ej., falta de tiempo durante la visita $(24,6 \%)$, falta de continuidad en las mismas $(12,1 \%)$. Es importante destacar que el 19,4\% considera como obstáculo la ausencia de protocolos específicos y que algunos profesionales mencionaron este hecho como una responsabilidad de la institución en el apartado "otros obstáculos". En este 
apartado también se reconoció cierto temor a las repercusiones, la falta de intervenciones específicas cuando los pacientes son identificados y la carencia de recursos en el entorno. Estos obstáculos más frecuentes son también hallados en otros estudios (Samuelson y Campbell, 2005; Thurston y Eisener, 2006).

No obstante, de manera significativa, un mayor porcentaje de médicos reconoce obstáculos en general y en todas las categorías propuestas excepto en la falta de continuidad en las visitas y la ausencia de protocolos. Es de destacar la diferencia hallada en la falta de tiempo como dificultad, un $63,6 \%$ de los médicos la perciben como tal, frente a un $5,9 \%$ de los psicólogos o un 22,2\% de los psiquiatras. Este hecho es un claro ejemplo de cómo la especificidad de la práctica habitual en los diferentes colectivos condiciona la realización de determinadas funciones propias de todos ellos.

Respecto a las intervenciones que realizan, la mayoría registra frecuentemente o siempre la declaración de la usuaria en su historia clínica (84\%) y le ofrecen información específica sobre $\operatorname{VCP}(67,1 \%)$. Por otra parte, la mayoría nunca o sólo a veces hacen intervenciones pautadas según un protocolo $(77,3 \%)$, derivan el caso a otro profesional $(60,2 \%)$ y proponen a la usuaria que denuncie $(51,1 \%)$.

Vemos que buena parte de los profesionales no contemplan la derivación, especialmente psiquiatras y psicólogos (el 71\% y el $68 \%$ respectivamente no lo proponen nunca o sólo a veces) frente a médicos (41\%). Estas cifras sorprenden si recordardamos que muchos de ellos no cuentan con un entrenamiento específico para la intervención en casos de VCP. En este sentido, hay que tener en cuenta la existencia de artículos de divulgación que abogan por la no derivación, y la consideran iatrogénica (Ramsay et. al 2002). Interesa replantear algunas creencias que dificultan la posibilidad de asistir por parte de profesionales especializados a víctimas de VCP. Respecto a la denuncia, los colectivos de psiquiatras y psicólogos son los que menos lo proponen a las usuarias $(33,3 \%$ y $41,8 \%$ respectivamente) frente al de médicos $(65,2 \%)$.

En relación al porcentaje de mujeres que sufren VCP en Cataluña cada año, éste se encuentra entre el
15 y el $30 \%$. Se advierte que dos terceras partes de los encuestados $(65,8 \%)$ subestiman esta cifra. No obstante, este hecho podría estar relacionado con un sesgo en la investigación, dado que no se incluyó una definición operativa concreta de VCP en la encuesta. Es probable, que si se hubiera explicitado el concepto (indicando los elementos que lo definen), el resultado pudiera ser otro.

\section{Conclusiones}

En el estudio hemos constatado la existencia de una gran sensibilidad entre los profesionales hacia el problema de la VCP y que éstos se enfrentan en su práctica a casos de esta índole pese a que no siempre exploran su presencia. Si a esto sumamos la consciente responsabilidad profesional, y el esfuerzo por la formación (aunque en general de forma autodidacta) nos encontramos en un contexto muy favorable para operar las modificaciones necesarias en la prevención de la violencia de género en el ámbito de la salud.

En general, se observa que, a pesar de las sugerencias de organismos e instituciones, la exploración no es rutinaria ni pautada y no se realiza durante la primera visita ni mediante preguntas directas, y depende en buena parte de la presencia de indicios. En este sentido, consideramos que el grado de conocimiento sobre el problema de la VCP y la actitud de muchos de los profesionales siguen siendo deficitarios.

Promover la sensibilización, una formación específica y actualizada, y la incorporación de indicaciones de cribado universal y de protocolos, pueden ser un paso decisivo para facilitar la tarea de detección de VCP. La planificación de estas acciones debe prestar atención además al tipo de obstáculos que cada colectivo de profesionales refiere y que dificulta su puesta en práctica.

Son necesarias más investigaciones dirigidas a evidenciar la efectividad de la aplicación de pruebas de cribado y al efecto de la formación especializada en las actitudes y prácticas de los profesionales; así como a la identificación de estrategias efectivas y a la adherencia al uso de guías de buenas prácticas. 


\section{Referencias}

Amor, P. J., Echeburúa, E., de Corral, P., Zubizarreta, I. y Sarasua, B. (2002). Repercusio-nes psicopatológicas de la violencia doméstica en la mujer en función de las circunstancias del maltrato. International Journal of Clinical and Health Psychology, 2, 227-246.

Andrés Pueyo, A. y Álvarez López, E. (2006). Detecció i prevenció de la violència domèstica contra les dones en l'atenció sanitària i l'assistència social. Barcelona: Documento interno de trabajo no publicado.

Cann, K., Withnell, S., Shakespeare, J., Doll, H. y Thomas, J. (2001). Domestic Violence: a comparative survey of levels of detection, knowledge and attitudes in healthcare workers. Public Health, 115, 89-95.

Coll-Vinent, B., Echevarría, T., Rodríguez, D. y Santiñá, M. (2007). Violencia intrafamiliar y de género vista por los profesionales de la salud. Medicina Clínica (Barcelona), 128, 317.

Comisión contra la Violencia de Género del Consejo Interterritorial del SNS. (2007). Protocolo común para la actuación sanitaria ante la violencia de género. Madrid: Ministerio de Sanidad y Consumo (Gobierno de España).

Elliot, L., Nerney, M., Jones, T. y Friedmann, P. (2002). Barriers to screening for domestic violence. Journal of General Internal Medicine, 17, 112-116.

Ellsberg, M. y Heise, L. (2005). Researching Violence against women: A practical guide for researchers and activist. Washington: World Health Organization, PATH.

Ellsberg, M., Jansen, H. A., Heise, L., Watts, C. H. y Garcia-Moreno, C. (2008). Intimate partner violence and women's physical and mental health in the WHO multi-country study on women's health and domestic violence: an observational study. Lancet, 371, 1165-1172.

García Moreno, C. (2002). Dilemmas and opportunities for an appropriate health-service response to violence aginst women. Lancet, 359, 15091514.

Guia per a l'abordatge del maltractament de gènere a l'atenció primària. (2003). Barcelona:
Institut Catalá de la Salut. Departament de Sanitat i Seguretat Social. Generalitat de Catalunya.

Ilustre Colegio Oficial de Médicos de Madrid. (2006). Al 78\% de las mujeres maltratadas no le habría importado que su médico le hubiera preguntado por su situación. Consultado febrero de 2008, disponible en http://www.icomem.es/noticias.php?do=extend\&idcont $=1020$

Krug, E. G., Dahlberg, L. L., Mercy, J. A., Zwi, A. B. y Lozano, R. (Eds.). (2002). World report on violence and health. Geneva: World Health Organization.

Lasheras Lozano, M. L. y Pires Alcaide, M. (Eds.). (2003). La violencia hacia las mujeres considerada como un problema de salud pública: Documento de apoyo para la atención a la salud de las mujeres víctimas. Documento técnico de Salud Pública, no86. Madrid: Servicio de Promoción de Salud. Instituto de Salud Pública.

Mc Farlane, J., Christoffel, K., Bateman, L., Miller, V. y Bullock, L. (1991). Assessing for abuse: Self-report versus nurse interview. Public Health Nursing, 8, 245-250.

Morrison, L. J., Allan, R. y Grunfeld, A. (2000). Improving the emergency department detection rate of domestic violence using direct questioning. Journal of Emergency Medicine, 19, 117124.

O.M.S. y O.P.S. (1998). Violencia contra la mujer: Un tema de salud prioritario. Washington: OMS/OPS.

Plazaola-Castaño, J. y Ruiz Pérez, I. (2004). Violencia contra la mujer en la pareja y consecuencias en la salud física y psíquica. Medicina Clínica (Barcelona), 122, 461-467.

Ramsay, J., Richardson, J., Carter, Y. H., Davidson, L. L. y Feder, G. (2002). Should health professionals screen women for domestic violence? Systematic Review. British Medical Journal, 325, 314-327.

Rodriguez, M. A., Bauer, H. M., McLoughlin, E. y Grumbach, K. (1999). Screening and Intervention for Intimate Partner Abuse: Practices and Attitudes of Primary Care Physicians. Journal of the American Medical Association, 282, 468-474.

Ruiz Pérez, I. y Plazaola Castaño, J. (2005). Intimate partner violence and mental health con- 
sequences in women attending family practice in Spain. Psychosomatic Medicine, 67, 791-797.

Samuelson, S. L. y Campbell, C. D. (2005). Screening for domestic violence: Recommendations based on a practice survey. Professional PsychologyResearch and Practice, 36, 276-282.

Stayton, C. D. y Duncan, M. M. (2005). Mutable influences on intimate partner abuse screening in health care settings: A synthesis of the literature. Trauma Violence and Abuse, 6, 271-285.

Thurston, W. E. y Eisener, A. C. (2006). Succseful integration and maintenance of screening for domestic violence in the health sector. Trauma Violence and Abuse, 7, 83-92.
Tomás Vecina, S., Chanovas Borrás, M. y Roqueta Egea, F. (2008). Violencia de género y servicios de urgencias. Medicina Clínica (Barcelona), 130, 278-279.

Vives Cases, C., Gil González, D., Carrasco Portiño, M. y Álvarez Dardet, C. (2006). Detección temprana de la violencia del compañero íntimo en el sector sanitario. ¿Una intervención basada en la evidencia? Medicina Clínica (Barcelona), 126, 101-104.

Wiist, W. H. y McFarlane, J. (1999). The Effectiveness of Abuse Assessement Protocol in Public Health Prenatal Clinics. American Journal of Public Health, 89, 1217-1221.

Artículo recibido: 21/04/2010

Revisión recibida: 26/04/2010

Artículo aceptado: 28/04/2010 